\title{
EXOGENOUS CAUSES OF MULTIPLE SCLEROSIS *
}

\author{
LEWELLYS F. BARKER, M.D. \\ BALTIMORE
}

My report has nothing to say concerning possib.e endogenous causes, and it also excludes certain exogenous etiologic agents such as direct bacterial infections or parasitic invasions, which are to be dealt with by others. The possible exogenous causes that I have to consider are: (1) the influence of earlier infections; (2) the influence of intoxications; (3) thermal and electrical influences, and (4) trauma.

With the aid of Dr. Caroline Latimer and Miss C. J. Smith, I have analyzed forty-four cases from my private practice and from the records of the Johns Hopkins Hospital, all studied between 1905 and 1921.

\section{INFECTIONS}

Pierre Marie of Paris has suggested, and the suggestion has been favorably entertained by various neurologists, that multip!e sclerosis might be a sequel of various infectious diseases. It has been observed in a certain number of .cases, not in many however, that the symptoms of multiple sclerosis began to appear soon after the occurrence of an infectious disease. Possibly in some of these cases it was a disseminated encephalomyelitis rather than a true multiple sclerosis that was dealt with. Granting, however, that occasionally true multiple sclerosis has occurred soon after an infection, this relationship may have been purely accidental. Unless the infection and the onset of multiple sclerosis are chronologically closely related, there could be little justification in considering the multiple sclerosis as a sequel of the infection. It has been fairly well established that multiple sclerosis may undergo exacerbation from intercurrent infection, and it seems probable that the instances in which multiple sclerosis has been supposed to begin after an infection have been instances in which the disease in reality existed unnoticed before the infection, its so-called "beginning" having been called forth by the infection.

If we take into account only cases in which an infection has preceded the apparent onset of multiple sclerosis by, say, two or three months, not longer, the statistics in the literature indicate that only from 3 to 5 per cent. of the cases recorded show such a temporal connection with infection (Berger; Hoffmann). In this country Woodberry (1919) has emphasized the co-existence of chronic tonsillar infections; Gill and Bassoe call attention to the frequency of both tonsillar and dental infections.

* Presented before the Association for Research in Nervous and Mental Diseases, Dec. 28, 1921. 
In the cases that have occurred in my own practice and in the Johns Hopkins Hospital, there was a history of one or another infection shortly before the onset of the symptoms of multiple sclerosis in five of the forty-four cases; these infections included gonorrhea (1), tonsillitis (1), nasopharyngitis and otitis (1), abscesses of the teeth (1), and influenza (1). This is a meager showing, and I attach no significance to it. In the distant past of the persons whose cases were analyzed, childhood infections were as common but no commoner than in other patients; five of the forty-four patients had had typhoid fever, five tonsillitis, five abscesses, four scarlet fever, two syphilis and one erysipelas. It will be seen that there is nothing striking about such a record of earlier infections.

Since the outbreak of epidemic encephalitis in 1917 the occurrence of a certain number of cases in which the disease became chronic, with exacerbation, has been noted, and doubtless many have wondered whether or not some of these might follow a course similar to that of true multiple sclerosis or possibly might even be identical with it. In the few necropsies that have been made on such cases, however, the lesions found have not been those of multiple sclerosis.

\section{INTOXICATIONS}

It was thought by Oppenheim that mineral poisoning, especially by lead, arsenic and tin, might be responsible for the origin of multiple sclerosis, and von Jaksch believed that poisoning by manganese could give rise to a clinical picture resembling multiple sclerosis if not identical with it. Undoubtedly multiple sclerosis occasionally occurs among workers in metals, and some of the European neurologists have drawn a part of their material from among such workers. But when we consider the total number of cases of multiple sclerosis, a history of poisoning by metals is rare among them, so rare that we can certainly exclude this form of poisoning as a principal etiologic agent. Multiple sclerosis is probably just as common in women as in men, or nearly so, and women are not exposed to metal poisoning. Again, in the majority of cases the disease begins in early life often at a period before the patients could have been subjected to metal poisoning from their occupations. Further, it is scarcely conceivable that the successive exacerbations that are characteristic of the disease can be explained on the ground of a succession of metal poisonings. In my series of fortyfour cases, a history of metal poisoning was found in none.

Other poisons incriminated include alcohol and carbon monoxid. Jelliffe mentions alcoholism in 8 per cent. of his cases, a larger incidence, however, than is found in most statistics. Among my forty-four cases there were only two patients who used alcohol to excess; the majority were total abstainers. When one recalls the enormous number of cases 
of alcoholism formerly seen and how rare it was to see multiple sclerosis in an alcoholic addict, it is improbable that it could be other than a predisposing or exacerbating factor. The same may be said of carbon monoxid poisoning. Disseminated lesions throughout the brain and spinal cord may occur in carbon monoxid poisoning, but these lesions do not appear to be identical with those of multiple sclerosis.

Dr. A. L. Skoog, of Kansas City, Mo., writes me that he has studied a painter who gave a positive history of intoxication by "volatile oils." None of our patients gave such a history.

In our Baltimore cases there were no intoxications of any sort recorded except intestinal stasis, and this feature was no more common than in the average of patients not suffering from multiple sclerosis.

\section{THERMAL AND ELECTRICAL INFLUENCES}

In some of the patients with multiple sclerosis one finds a history of heat stroke, of lightning stroke or of exposure to cold and wet. But such instances are rare, and the hypotheses that have been put forward to explain them (reflex contraction of vessels in the central nervous system due to cold; chemical changes in hemoglobin brought about by thermal influences) seem forced. Krafft-Ebing reported thermic injury in no less than forty of 100 patients. Otto Marburg saw a patient whose clothing, suddenly wet, froze on him; six days later he began to have visual disturbances due to retrobulbar neuritis, after which the typical picture of multiple sclerosis developed. Most critical observers express the opinion, however, that these thermal influences are not the causes of multiple sclerosis but, at most, are injuries that cause an exacerbation of an already existing disease. In our fortyfour cases, the analysis does not reveal a single instance in which there had been electrical injuries, thermal injuries, or marked exposure to cold or wet.

\section{TRAUMATISM}

Patients with chronic nervous disease are prone to incriminate trauma, physical or psychic, as causative agent. Similarly, multiple sclerosis is not infrequently attributed to traumatic causes. It has been assumed that the trauma injures the blood vessels, does harm by violent commotion in the cerebrospinal fluid or by concussion causing molecular changes in the nerve substance, or by tearing lymph spaces with resulting necrosis in the parenchyma. No one has, however, been able to reproduce the lesions of multiple sclerosis through experimental traumatism to animals. If one adheres to the requirements laid down by $\mathrm{K}$. Mendel, the incidence is small. Mendel laid down as necessary conditions: (1) direct injury to the skull or spine or violent shaking of the same; (2) exclusion of every other cause; (3) demonstration of 
complete health before the trauma, and (4) demonstration of a definite temporal connection between the appearance of the first symptoms of the disease and the accident. The incidence when these conditions are regarded varies according to different authors-Schultze, 8 per cent.; Berger and O. Marburg, 9 per cent.; Jelliffe, 12 per cent.

It must be remembered that injuries often occur in multiple sclerosis as a result, rather than as a cause, of the disease, and when multiple sclerosis appears to develop after trauma careful inquiry will often show that symptoms of the disease had been present earlier. It does seem certain, however, that latent multiple sclerosis may suddenly become manifest after a severe trauma (either physical or psychic) and that manifest multiple sclerosis may undergo marked exacerbation after trauma.

In our forty-four cases, eleven patients gave a history of physical trauma of one or another sort, and three gave a history of nervous and mental strain. In most of the instances, however, the trauma had occurred long before the onset of the illness; in only three cases did it occur within a year of onset. Three of the female patients stated that the onset and progress of the disease seemed to be related to childbearing.

\section{CONCLUSIONS}

If multiple sclerosis is a disease entity due to a single cause that acts in early life, it may be due to some specific infection, but the evidence available is strongly against its being caused by any of our wellknown infections, by any ordinary intoxication (organic or inorganic), or by electrical, thermal or traumatic influences. If the exogenous factors mentioned play any rôle at all in the etiology of the disease, they must act either as predisposing influences for the true cause or as aggravators of a disease already started by the true cause. 